\title{
A Markov Chain Approximation to Choice Modeling
}

\section{Introduction}

Assortment optimization is an important problem that arises in many industries such as retailing and airlines where the decision maker needs to decide on an optimal subset of products to offer to maximize the expected revenue. The demand and the revenue of any product depends on the complete set of offered products since customers potentially substitute to an available product if their most preferred product is not available. Such a substitution behavior is captured by a customer choice model that can be thought of as a distribution over preference lists (or permutations of products). A customer with a particular preference list purchases the most preferable product that is available. Therefore, the choice model specifies the probability that a customer selects a particular product for every offer set. One of the key challenges of any assortment planning problem is to find the "right choice model" to describe the substitution behavior when we only observe historical sales data for a small number of assortments. The underlying customer preferences are latent and unobservable.

A choice model, in the most general setting, can be thought of as a distribution over permutations that arise from preferences. In the random utility model of preferences, each customer has a utility that depends on the attributes of the product and a random idiosyncratic component, i.i.d according to some distribution. The preference list of the customer is given by the decreasing order of utilities of products. This model was introduced by Thurstone [7] in the early 1900s. An important special case of the above model is obtained assuming idiosyncratic components are i.i.d according to an extreme value distribution such as Gumbel. This model also referred to as the Plackett-Luce model and was proposed independently by Luce [2] and Plackett [4] and later McFadden [3] referred to it as a Multinomial logit model. The MNL model is by far the most popular model as both the estimation as well as the optimization problems are tractable for this model (see [6]). However, the MNL model is not able to capture heterogeneity in substitution behavior and also suffers from the Independence from Irrelevant Alternatives (IIA) property which limit the applicability of the MNL model.

More complex choice models such as the Nested logit model and the mixture of MNL models have been studied in the literature. However, both the estimation and the resulting optimization problem become difficult when we use a richer class of parametric models. Rusmevichientong et al. [5] show that the assortment optimization problem is NP-hard for a mixture of MNL model even for the case of mixture of only two MNL models. The work by Farias et al. [1] and van Ryzin and Vulcano [8] are most closely related to our work. Farias et al. [1] consider a non-parametric approach where they use the distribution over permutations with the sparsest support that is consistent with the data. However, the resulting assortment optimization problem can not be solved efficiently for the sparsest support distribution. In van Ryzin and Vulcano [8], the authors consider an iterative expectation maximization algorithm to learn a non-parametric choice model 
where in each iteration they add a new MNL to the mixture model. However, optimization over mixture of MNLs is NP-hard [5].

\section{Our Contributions}

We present a new tractable data-driven approach to choice modeling that is robust to model selection errors. Our approach is based on a new primitive to model the substitution behavior. The substitution behavior of any customer is captured by his preference list. The substitution behavior can be interpreted as sequential transitions from one product to another in the order defined by the preference list until the customer finds an available product.

Markov Chain based Choice Model. Motivated by the above interpretation, we present a new choice model where substitution behavior is modeled as a sequence of state transitions of a Markov chain where there is a state for each product including the no-purchase alternative. We model the substitution behavior as follows: a customer arrives in the state corresponding to his most preferable product. If that product is not available, he/she transitions to other product states according to the transition probabilities of the Markov chain. Therefore, the sequential transitions based on the preference list are approximated by Markovian transitions in the Markov chain based choice model.

The Markov chain based choice model is completely specified by the arrival probabilities in each state and the transition probability matrix. We show that both the arrival probabilities to each state and the transition matrix can be estimated efficiently from choice probability data for a small number of assortments. Furthermore, given the arrival probabilities and the transition probabilities, we can efficiently compute the choice probabilities for all assortments for the Markovian substitution model. For any assortment, we modify the Markov chain to make all states corresponding to products in the offer set as absorbing. Then the stationary distribution over all absorbing states (including the no-purchase alternative) gives us the choice probabilities of all products. These can be computed efficiently by solving a system of linear equations.

Approximation Bounds. We show that the Markov chain choice model provides a good approximation to any random utility discrete choice models under mild assumptions. The class of models arising from a random utility model is quite general and includes all models that can be expressed as distributions over permutations. This class includes MNL, Nested logit (NL) and mixture of MNL (MMNL) models. We present lower and upper bounds, related to the spectral properties of the Markov chain, on the ratio of the choice probability computed by the Markov chain model and the true underlying model. These bounds show that the Markov chain model provides a good approximation for all random utility based choice models under very mild assumptions.

Furthermore, we show that the Markov chain model is exact if the underlying model is MNL. In other words, if the the Markov chain model parameters are estimated from data from an underlying MNL model, then the choice probability computed by the Markov chain model coincides with the probability given by the MNL model for all products and all assortments. We would like to emphasize that the estimation of the Markov chain is data-driven and does not require any knowledge about the type of underlying model that generates the data. Therefore, the Markov chain model circumvents the challenging model selection problem for choice modeling and provides a simultaneous approximation for all discrete choice models. 
Computational Study and Asymptotic Bounds. In addition to the theoretical approximation bounds, we present a computational study to compare the choice probability estimates of the Markov chain model as compared with the choice probability of the true model. In particular, we consider random instances of mixture of MNL models and compare out of sample performance of the Markov chain model with respect to the true mixture of MNL model. The numerical experiments show that our model performs extremely well on random instances of mixture of MNLs. In particular, the maximum relative error of choice probabilities estimates as compared to the true choice probabilities is less than $3 \%$ on average.

Assortment Optimization. We show that the assortment optimization problem can be solved optimally in polynomial time for the Markov chain choice model. In an assortment optimization problem, the goal is to find an assortment (or offer set) that maximizes the total expected revenue. This result is quite surprising since in the Markov chain based choice model, we can not even express the choice probability as a simple functional form of the model parameters. Therefore, we are not able to even formulate the assortment optimization problem as a mathematical program directly. However, we present a policy iteration algorithm to compute an optimal assortment in polynomial time for the Markov chain based choice model. Moreover, our algorithm shows that the optimal assortment is independent of the arrival rates. This provides interesting insights about the structure of the optimal assortment. Furthermore, we show that if Markov chain model is estimated from data from an underlying latent choice model, then the optimal assortment for the Markov chain model is a good approximation for the assortment optimization problem over the underlying latent model.

\section{References}

[1] V.F. Farias, S. Jagabathula, and D. Shah. A nonparametric approach to modeling choice with limited data. Management Science (To Appear), 2012.

[2] R.D. Luce. Individual choice behavior: A theoretical analysis. Wiley, 1959.

[3] D. McFadden. Conditional logit analysis of qualitative choice behavior. in P. Zarembka, ed., Frontiers in Econometrics, 1973.

[4] R.L. Plackett. The analysis of permutations. Applied Statistics, pages 193-202, 1975.

[5] P. Rusmevichientong, D. Shmoys, and H. Topaloglu. Assortment optimization with mixtures of logits. Technical report, Tech. rep., School of IEOR, Cornell University, 2010.

[6] K. Talluri and G. Van Ryzin. Revenue management under a general discrete choice model of consumer behavior. Management Science, 50(1):15-33, 2004.

[7] L.L. Thurstone. A law of comparative judgment. Psychological Review; Psychological Review, 34(4):273, 1927.

[8] G. van Ryzin and G. Vulcano. An expectation-maximization algorithm to estimate a general class of non-parametric choice-models. Technical report, working paper, 2011. 